\title{
Epsilon regime calculations with reweighted clover fermions
}

\author{
Anna Hasenfratz* \\ Department of Physics, University of Colorado, Boulder, Colorado-80309-390,USA \\ E-mail: annaleotvos.colorado.edu
}

Roland Hoffmann

Bergische Universität Wuppertal, Gaussstraße 20, 42219 Wuppertal, Germany

hoffmannepizero.colorado.edu

\section{Stefan Schaefer}

Institut für Physik, Humboldt Universität, Newtonstraße 15, 12489 Berlin, Germany

s.chaef@physik.hu-berlin.de

\begin{abstract}
We perform fully dynamical simulations at small quark masses by reweighting in the quark mass, calculating the weight factors stochastically. This approach avoids some of the technical difficulties associated with direct simulations. We find that the weight factors fluctuate only moderately on nHYP smeared dynamical Wilson-clover ensembles, and demonstrate that the overlap between the original and reweighted configurations is large both for short and long distance observables. We could successfully reweight $16^{4},(1.85 \mathrm{fm})^{4}$ volume configurations from $m_{q} \approx 20 \mathrm{MeV}$ to $m_{q} \approx 5 \mathrm{MeV}$, and $24^{4},(2.77 \mathrm{fm})^{4}$ configurations from $m_{q} \approx 8 \mathrm{MeV}$ to $m_{q} \approx 3 \mathrm{MeV}$ quark masses, reaching the $\varepsilon$-regime. Using the pseudoscalar and axialvector correlators we predict the low energy constants $\Sigma$ and $F$ and study their volume and mass dependence.
\end{abstract}

The XXVI International Symposium on Lattice Field Theory

July 14 - 19, 2008

Williamsburg, Virginia, USA

\footnotetext{
*Speaker.
} 


\section{Introduction}

With efficient simulation techniques, improved lattice actions and increased computational resources, essentially all parameter values of lattice QCD, including the point of physical quark masses, are accessible to direct simulation today. However, in the small quark mass regime the challenges are still considerable:

- Large volumes are needed for the stability of the algorithms when Wilson fermions are used

- Autocorrelation times increase dramatically towards the chiral limit

- Statistical fluctuations of fermionic correlators become difficult to estimate since configurations with large contributions become rare as small Dirac modes are more and more suppressed.

A possible solution to counter these problems is to avoid generating an ensemble with the fermionic weight of the light target quark mass but instead simulate a heavier quark mass and reweight to the desired ensemble. Simulational algorithms are more efficient at the larger mass and smaller volumes are sufficient from the algorithmic point of view, while the autocorrelation time is controlled by the heavier simulational quark mass. At a larger quark mass the region of small Dirac eigenvalues is oversampled with respect to the target distribution, thus observables that receive large contributions there (e.g. pseudoscalar correlators) will be better estimated.

On the other hand reweighting will fail if the overlap between the generated and target configuration ensembles is small, or if the weight factors fluctuate strongly. One also has to overcome the computational problem of calculating the weight factors effectively. In two recent papers $[1,2]$ we have developed a technique that solves the latter problem and demonstrated that reweighting is possible and efficient on fairly large volumes between significantly different quark masses. We have applied the technique to reach the $\varepsilon$-regime with Wilson fermions and predicted the values of the low energy constants $\Sigma$ and $F$. In this paper we summarize the technique, giving further supportive evidence for the validity of reweighting. We also briefly discuss the $\varepsilon$-regime analysis, including the use of Wilson fermions in the $\varepsilon$-regime and the validity of the continuum chiral perturbation theory in our analysis.

An alternative approach to reweighting was presented in this conference [3].

\section{Reweighting in the quark mass}

The numerical simulations for this project were done with 2 flavors of nHYP smeared Wilsonclover fermions and one-loop Symanzik improved gauge action[4]. We use the tree-level $c_{S W}=1.0$ clover coefficient, so our action is not fully $\mathscr{O}(a)$ improved, but we expect that the $\mathscr{O}(a)$ corrections are small. We have generated two sets of gauge ensembles, both at gauge coupling $\beta=7.2$. The first set consists $180,16^{4}$ configurations at $\kappa=0.1278$, the second $154,24^{4}$ configurations at $\kappa=0.12805$.

We set the lattice scale from the static quark potential, using $r_{0}=0.49 \mathrm{fm}$ for the Sommer parameter. On both configuration sets we found $r_{0} / a=4.25(2)$, giving $a=0.1153(5) \mathrm{fm}$. With 

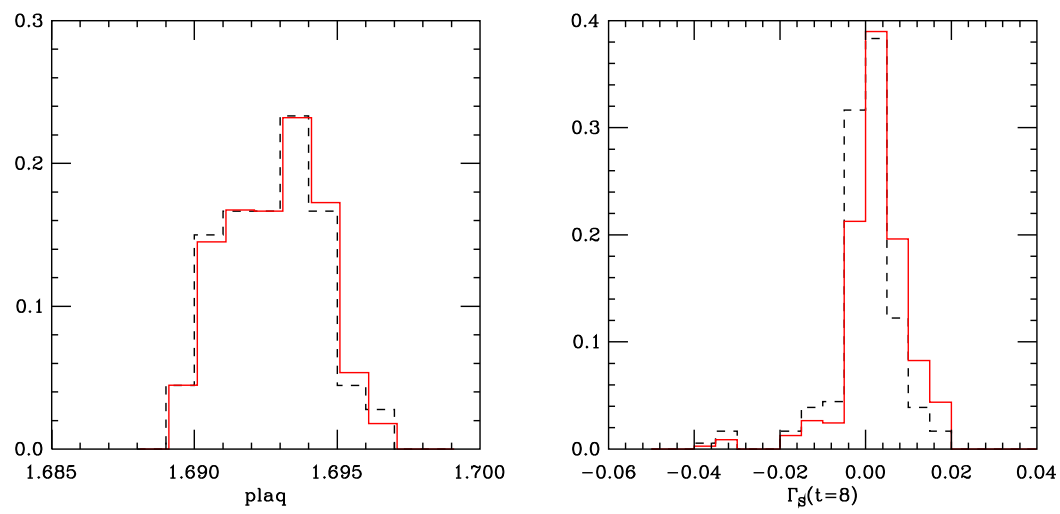

Figure 1: The distribution of the plaquette (left panel) and scalar correlator at $t=8$ (right panel) on the $16^{4}$ ensemble at $\kappa=0.1280$. On both panels the dashed line is the original (partially quenched) distribution and the solid lines correspond to the reweighted distributions. The reweighting included the $\beta_{\mathrm{nHYP}}$ term as explained in the text.

this value the physical volumes are $(1.85 \mathrm{fm})^{4}$ and $(2.77 \mathrm{fm})^{4}$. We connect the PCAC quark mass to the renormalized one using the pseudoscalar and axialvector renormalization factors obtained with the RI-MOM method, $Z_{P}^{\overline{M S}}=0.90(2), Z_{A}^{\overline{M S}}=0.99(2)$, and estimate the renormalized quark mass in the $\overline{\mathrm{MS}}$ scheme at $2 \mathrm{GeV}$ to be 22 and $8.5 \mathrm{MeV}$ on the 2 configuration sets, respectively.

Starting from the original configurations one can explore a range of quark masses in fully dynamical systems by reweighting the configurations. The necessary weight factor is the ratio of the fermion determinants that can be calculated stochastically, but one must take care not to introduce significant statistical errors with the stochastic process. We apply three methods, low mode separation, determinant breakup, and ultraviolet (UV) noise reduction to control the statistical fluctuations. In both the $16^{4}$ and $24^{4}$ ensembles we separate 6 low Hermitian eigenmodes. In addition we break up the determinant to the product of 33 and 60 terms for each $\Delta \kappa=0.0001 \mathrm{shift}$ in reweighting on the $16^{4}$ and $24^{4}$ volumes, respectively.

To control and remove some of the UV noise we introduce an nHYP plaquette pure gauge term in the reweighted action with coefficient $\beta_{\mathrm{nHYP}}=6.0\left(\kappa-\kappa_{\text {rew }}\right)$. This value is so small that within errors we do not observe its effect on the lattice spacings or quark masses. It is worth emphasizing that including the nHYP plaquette gives simply an alternative gauge action and it does not introduce any systematical errors.

With the above outlined method we could reweight the $16^{4}$ configurations from $\kappa=0.1278$ to $\kappa=0.1281$, or approximately $5 \mathrm{MeV}$ quark mass, and the $24^{4}$ configurations from $\kappa=0.12805$ to $\kappa=0.12815$, or $3.8 \mathrm{MeV}$ quarks. The calculation automatically gives the weight factors at inbetween $\kappa$ values and we analyzed both data sets at 4 different masses.

In addition to removing the UV fluctuations and thus reducing the fluctuations of the weight factors, the introduction of the nHYP plaquette term also increases the overlap between the original and target ensembles. The largest weight factors are pushed from the edge of the plaquette distribution to the middle, where the statistical sampling is better, and the effect is similar for other observables as well. We illustrate this in Figure 1 with the distributions of the plaquette and the scalar correlator at $t=8$. All data correspond to the $16^{4}$ data set at $\kappa=0.1280(\approx 9 \mathrm{MeV}$ quarks $)$. 


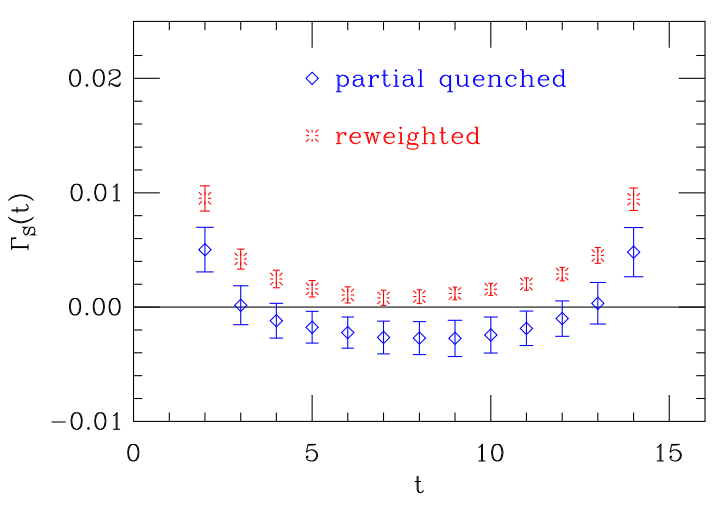

Figure 2: The scalar correlator at $\kappa=0.1280$ for the partially quenched and for the reweighted ensembles.

For both quantities the overlap between the reweighted and original distributions is excellent, there is no sign that reweighting would prefer region that is poorly sampled by the original ensemble. The main difference between the reweighted and partially quenched scalar data is the suppression of the negative contribution of the latter one. This seemingly slight difference is nevertheless sufficient to make the scalar correlator positive. In Figure 2 we compare the partially quenched and reweighted, thus fully dynamical, scalar correlators. Not only does the reweighted correlator stay positive, its statistical errors are also significantly reduced compared to the partially quenched one. The overlap between the reweighted and partially quenched correlators for other mesons are similar, typically better, than for the scalar one.

\section{3. $\varepsilon$-regime analysis}

In the $\varepsilon$-regime the pion correlation length is large compared to the linear size of the lattice, the light pseudoscalar mesons dominate the dynamics. Nevertheless, in order to incorporate the massive modes the volume has to be large compared to the QCD scale, or $L \gg F$. Chiral perturbation theory predicts that at next-to-leading (NLO) order the meson correlators are quadratic in time and depend only on two low-energy constants, $\Sigma=\lim _{m \rightarrow 0}\langle\bar{q} q\rangle$ and $F=\lim _{m \rightarrow 0} F_{\pi}$. The explicit formulas have been calculated for the different meson correlators in continuum $\chi P T$, averaged over topological sectors, in [5, 6]. In the case of the Wilson lattice action one expects extra terms in the chiral expansion, but they show up only at next-to-next-to-leading order, so as long as the NLO formulas describe the data, the continuum expressions are sufficient.

An important question to consider if Wilson fermions can be used at all in the $\varepsilon$-regime. As the quark mass decreases in a large volume (p-regime) simulation, the chiral symmetry breaking effects of Wilson fermions get large compared to the mass, and that can create large lattice artifacts. In practice the continuum limit has to be taken before the chiral limit. The situation is different in the $\varepsilon$-regime, where the finite volume of the system creates an infrared cutoff even at vanishing quark mass. This effect is well illustrated by the Hermitian gap distribution. While in infinite volume one expects the median of the gap to scale with the mass $\bar{\mu}=Z_{A} m_{\text {PCAC }}$ [7], in the $\varepsilon$-regime $\bar{\mu}$, governed by the IR cutoff of the volume, remains finite while $m_{\mathrm{PCAC}} \rightarrow 0$. This is clearly the case in our simulations. While the quark mass changes by a factor of 4 as we reweight from $\kappa=0.1278$ 

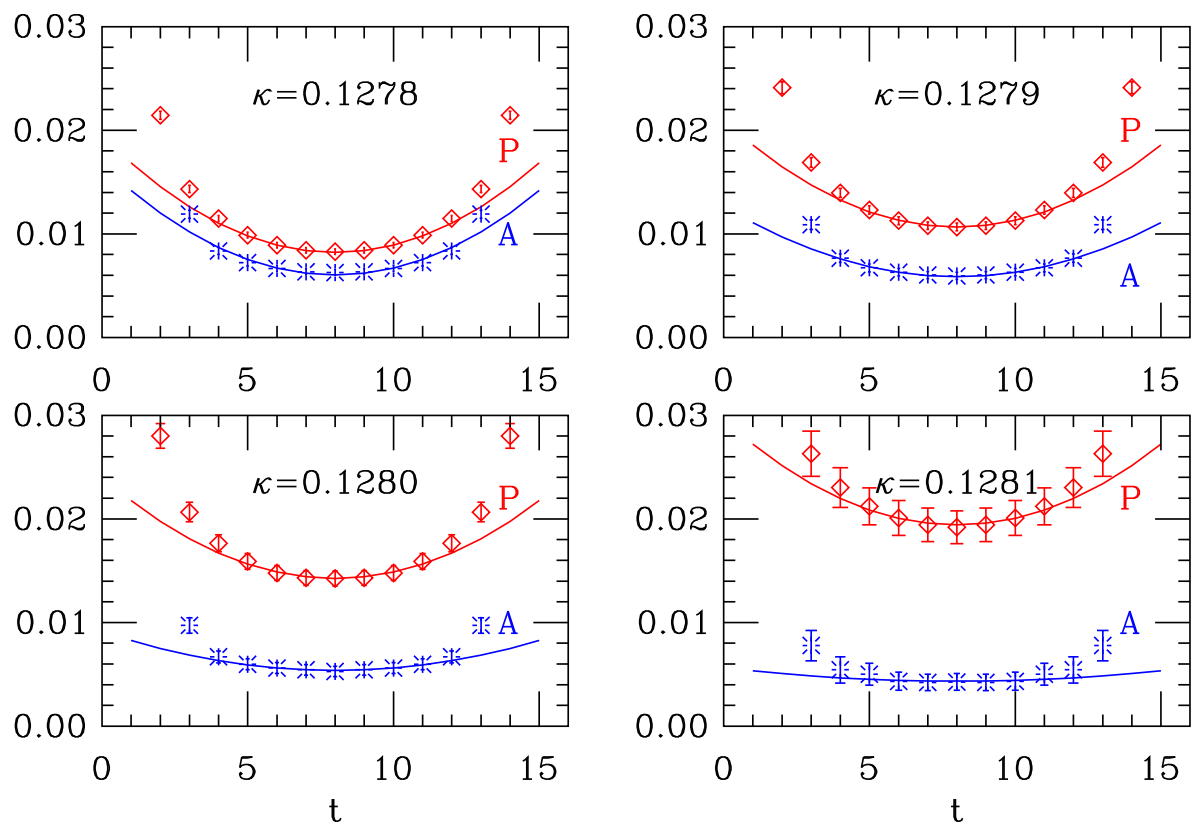

Figure 3: The pseudoscalar ( red diamonds) and axialvector (blue bursts) lattice correlators and the combined fit results on the $16^{4}$ data set. The axialvector correlators are multiplied by the factor 50 to better match the scale of the pseudoscalar.

to $\kappa=0.1281$ on the $16^{4}$ ensemble, the median of the gap changes by about $40 \%$ only [2]. One does not need a chiral action to study the epsilon regime, though the explicit symmetry breaking effects should be small compared to the inverse lattice size.

In this study we consider the pseudoscalar and the axialvector correlators. The former one is dominated by $\Sigma$, the latter by $F$, though both depend on the $\mathscr{O}(1)$ combination $m \Sigma V$ and the $\mathscr{O}\left(\varepsilon^{2}\right)$ quantity $1 /(F L)^{2}$. The lattice correlators have to be multiplied by the renormalization factors $Z_{P}^{2}$ and $Z_{A}^{2}$ to obtain the continuum ones, while in the product $m \Sigma V$ the quark mass can be expressed in terms of the PCAC mass as $m=m_{\mathrm{PCAC}} Z_{A} / Z_{P}$. In our analysis we do a combined fit to the continuum pseudoscalar and axialvector correlators.

The $\varepsilon$-expansion formulas are systematic expansions in the parameter $1 /(F L)^{2}=\mathscr{O}\left(\varepsilon^{2}\right)$, but depend on the $\mathscr{O}(1)$ quantity $m \Sigma V$. In our simulation we explore the range $m \Sigma V \approx 0.7-5.0$. Large values introduce large NLO and NNLO corrections to the correlators, and at some point one transitions into the large volume $p$-regime. Only by examining the fit results can we decide what range of $m \Sigma V$ values are acceptable in the $\varepsilon$-regime. The volume dependence is also an important issue to consider. On our smaller, $(1.87 \mathrm{fm})^{4}$ volume, the $\varepsilon$-regime expansion parameter is rather large, $1 /(F L)^{2} \approx 1.45$, and one expects large corrections to the NLO formulas. On our larger volume $1 /(F L)^{2} \approx 0.46$, the NLO expansion is much more reliable.

Our philosophy is to fit both the small and large volume data with the NLO $\chi P T$ formulas and study the quality of the fit and the change of the predicted low energy constants as the volume increases and quark mass decreases.

The fits on the $16^{4}$ lattices are shown in Figure 3, where we plot both the pseudoscalar and 

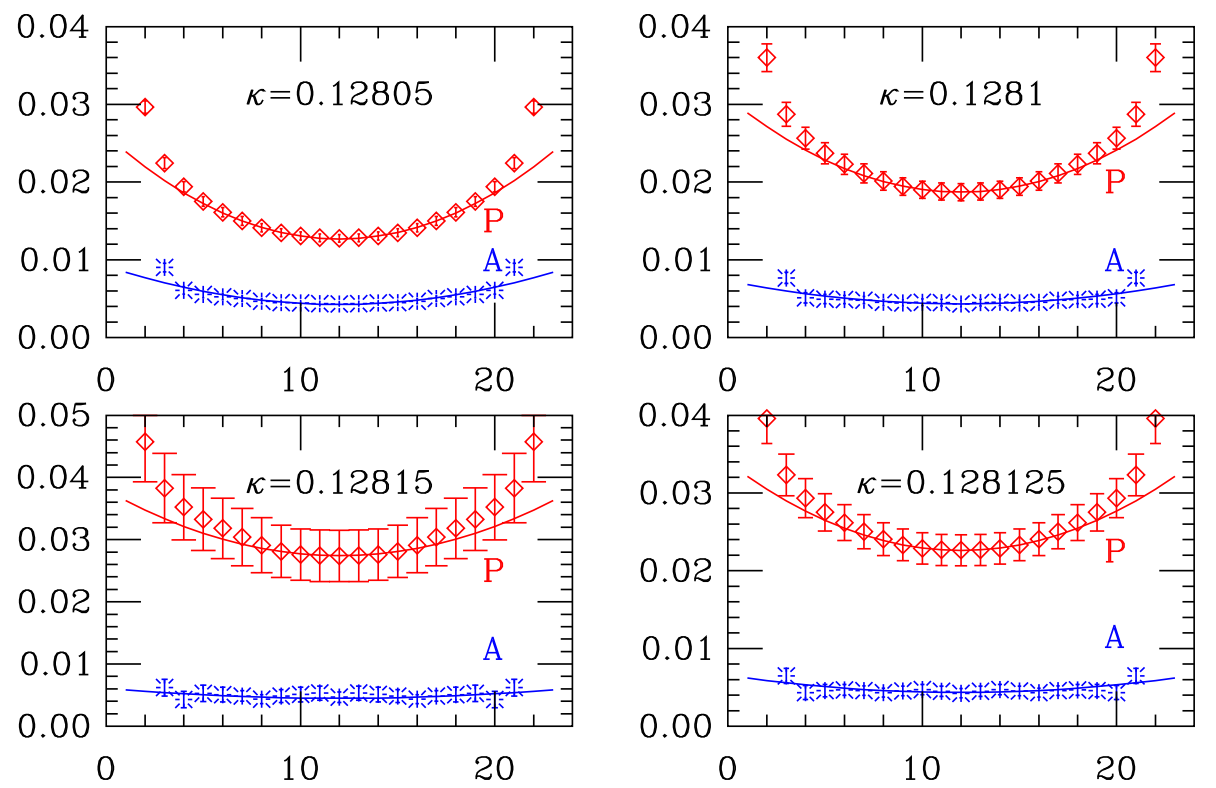

Figure 4: Same as Figure 3 but on the $24^{4}$ data set .
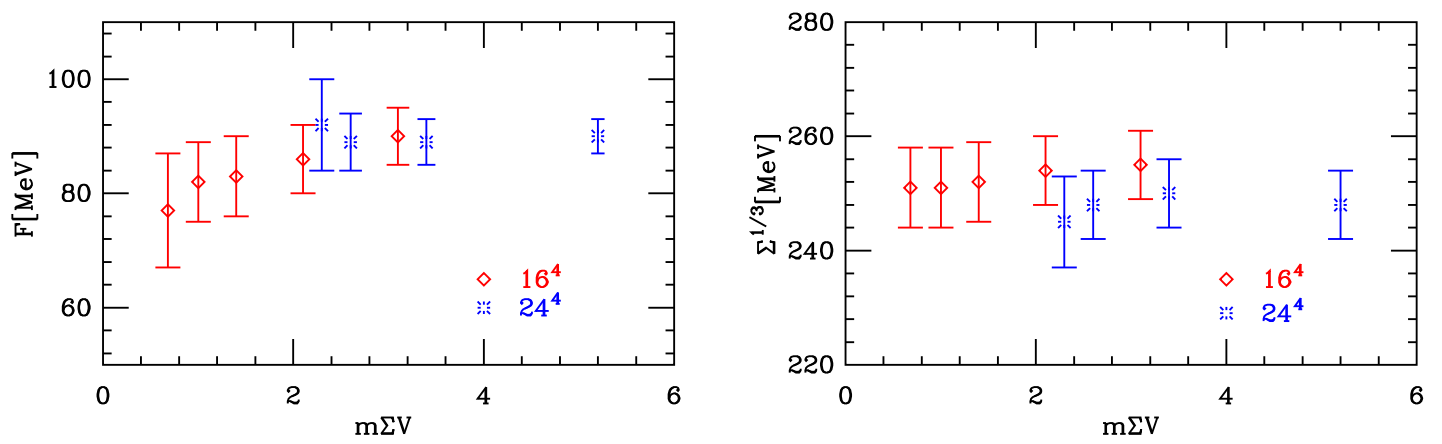

Figure 5: The low-energy constant $F$ and $\Sigma^{1 / 3}$ as the function of the parameter $m \Sigma V$, predicted by NLO $\chi$ PT.

axialvector correlators. The data are well described by the NLO formulas at all four mass values. Figure 5 shows the predictions for $F$ and $\Sigma$ as the function of $m \Sigma V$. While the condensate $\Sigma$ is basically constant, $F$ shows a slight drift as the quark mass decreases.

Our second data set is $24^{4},(2.77 \mathrm{fm})^{4}$, considerably larger. Figure 4 shows the combined fit and, again, we find good agreement for all correlators. The statistical errors are under control everywhere, though they increase as the reweighting range increases .

The low energy constant $\Sigma$ on the larger volume is consistent with the value obtained on the small volume (see Figure 5). In case of $F$ the large volume data is independent of the quark mass and is consistent with the small volume data where the two sets overlap. We cannot exclude the possibility that in the large volume data we see the breakdown of the $\varepsilon$-expansion at large $m \Sigma V$, but we believe finite volume corrections are more likely to explain the difference. Since the next-toleading order corrections to $F$ are over $10 \%$ on the $16^{4}$ data set, we prefer using the large volume 
data to arrive at our final prediction,

$$
F=90(4) \mathrm{MeV}, \quad \Sigma^{1 / 3}=248(6) \mathrm{MeV} .
$$

The errors only include the statistical uncertainties. These values are consistent with other recent two-flavor computationsi $[8,9]$.

\section{Conclusion}

The data presented in this paper have been generated with moderate computer resources. This was possible due to the good chiral properties of the action which come at relatively low cost due to the simple nHYP smearing procedure, and the effective reweighting that allowed us to lower the quark mass even further. We have demonstrated good overlap for different observables between the original and reweighted data sets.

With reweighting we were able to reach the $\varepsilon$-regime with Wilson fermions on 2 different volumes. Since the $\chi$ PT expansion converges slowly in $1 /(F L)^{2}$, our large volume puts us into a good position and the comparison between the $L / a=16$ and $L / a=24$ results shows that the finite volume effects are under control.

For the $\varepsilon$ expansion to be valid, the parameter $m \Sigma V$ has to be $\mathscr{O}(1)$. Our data span the range 0.7 to 5.2 and might go beyond the validity of the analytical expressions. An expansion that connects the $\varepsilon$ and $p$ regimes would be very useful to control this aspect of the calculation.

Repeating the calculation at a smaller lattice spacing would not be prohibitively expensive and could improve on all of the above mentioned issues.

\section{Acknowledgement}

Most of the numerical work reported in this paper was carried out at the kaon cluster on FNAL. We acknowledge the support of the USQCD/SciDac. This research was partially supported by the US Department of Energy and the Deutsche Forschungsgemeinschaft in the SFB/TR 09.

\section{References}

[1] A. Hasenfratz, R. Hoffmann and S. Schaefer, Phys. Rev. D78 (2008) 014515 [0 805 . 2369 ].

[2] A. Hasenfratz, R. Hoffmann and S. Schaefer, Phys. Rev. D78 (2008) 054511 [0 806.4586 ].

[3] P. Palombo and M. Luscher, Contribution at this conference.

[4] A. Hasenfratz, R. Hoffmann and S. Schaefer, JHEP 05 (2007) 029 [hep-lat/ 0702028 ].

[5] P. Hasenfratz and H. Leutwyler, Nucl. Phys. B343 (1990) 241-284.

[6] F. C. Hansen and H. Leutwyler, Nucl. Phys. B350 (1991) 201-227.

[7] L. Del Debbio, L. Giusti, M. Lüscher, R. Petronzio and N. Tantalo, JHEP 02 (2006) 011 [hep-lat/0512021].

[8] JLQCD Collaboration, H. Fukaya et. al., 0711.4965.

[9] K. Jansen, A. Nube, A. Shindler, C. Urbach and U. Wenger, PoS LATTICE2007 (2007) 084 [0711.1871]. 